\title{
Philonsorbonne
}

4 | 2010

Année 2009-2010

\section{Le philosophe et l'écrivain : nature du discours philosophique chez Gilles Deleuze}

\section{Axel CHERNIAVSKY}

\section{(2) OpenEdition}

1 Journals

Édition électronique

URL : https://journals.openedition.org/philonsorbonne/251

DOI : 10.4000/philonsorbonne.251

ISSN : $2270-7336$

Éditeur

Publications de la Sorbonne

\section{Édition imprimée}

Date de publication : 15 mai 2010

Pagination : $9-29$

ISBN : 978-2-85944-647-5

ISSN : 1255-183X

\section{Référence électronique}

Axel CHERNIAVSKY, « Le philosophe et l'écrivain : nature du discours philosophique chez Gilles Deleuze », Philonsorbonne [En ligne], 4 | 2010, mis en ligne le 31 janvier 2013, consulté le 11 juin 2021. URL : http://journals.openedition.org/philonsorbonne/251 ; DOI : https://doi.org/10.4000/ philonsorbonne.251 


\title{
Le philosophe et l'écrivain : nature du discours philosophique chez Gilles Deleuze
}

\author{
Axel Cherniavsky
}

La philosophie est définie par Gilles Deleuze comme une création de concepts $^{1}$. Elle implique l'invention d'une écriture et comporte une part inévitable de fiction. Elle s'apparente ainsi à l'art littéraire et au travail de l'écrivain. Deleuze lui-même revendique cette filiation : «Je ne sais pas (...) si je me considère comme un écrivain en philosophie, déclare-t-il ; je sais que tout grand philosophe est un grand écrivain $»^{2}$. Dès lors, se pose la question de savoir en quoi la philosophie est une forme de littérature et en quoi consiste exactement la création de concepts. Cette thèse se heurte en effet à l'idée communément répandue selon laquelle les philosophes écrivent mal et à son présupposé, à savoir que le discours philosophique aurait la place d'un ornement accessoire, ou le rôle d'une mise en forme. Il s'agit alors de comprendre comment la création conceptuelle exige un travail sur le langage et repose sur une théorie du discours et une philosophie de l'art littéraire. Or à partir du moment où le concept motive un travail littéraire et où la théorie du discours philosophique s'enracine dans une philosophie de l'art littéraire, un nouveau problème semble s'ajouter au premier. $\mathrm{Ne}$ serions-nous pas dans le cadre d'un esthétisme ? Le soupçon s'approfondit lorsqu'on se souvient que la réflexion se développe au sein d'une pensée qui comprend la philosophie comme une création voire comme un art, l'histoire de la philosophie comme théâtre ${ }^{3}$, collage ${ }^{4}$ ou art du portrait philosophique, et pour laquelle certains éléments constituants d'un système philosophique sont des personnages conceptuels ${ }^{6}$. Quelle différence y a-t-il entre cette thèse et une déclaration comme celle de Borges, selon laquelle la philosophie

\footnotetext{
1. $Q P h$, p. 10. Le lecteur trouvera la liste des abréviations à la fin de cet article, p. 29.

2. $A B C$, Lettre L ; 2:04:20-2:04:30.

3. ID, p. 199.

4. $D R$, p. 4.

5. $Q P h$, p. 55 ; $P P$, p. $185-186$.

6. $Q P h$, p. 60-81.
} 
est une «branche de la littérature fantastique ${ }^{7}$, déclaration qui renferme tout son scepticisme, épistémologique et éthique, et qui n'assigne à la philosophie que le lieu d'une activité ludique ? Est-ce à dire que la philosophie se ramène à une pure fiction gratuite et doive renoncer à toute prétention à la vérité ? Toute la question est de savoir quelle est au juste la nature de cette création de concepts qui définit l'activité philosophique. Pour cela, il est nécessaire à la fois d'éclairer sa parenté avec le travail littéraire et de dégager sa spécificité. C'est pourquoi il s'agira d'abord d'examiner les principales caractéristiques de la littérature chez Deleuze pour voir ensuite en quoi la philosophie les vérifie et s'en distingue par un travail d'écriture qui lui est propre. Dans cette optique, il conviendra de procéder à une analyse du style propre à Deleuze. C'est en effet dans sa propre écriture que l'on pourra observer concrètement comment la création de concepts a lieu dans le langage et dissiper les soupçons d'esthétisme en montrant les fonctions propres à l'énonciation philosophique.

\section{Nature de la littérature et de ses fonctions}

Si pour devenir un grand philosophe, il faut être un grand écrivain, il importe de bien cerner les traits caractéristiques de l'écriture littéraire et d'en saisir les fonctions. La littérature se présente d'abord comme une mise en variation de la langue, comme une utilisation singulière. C'est dans ce sens que la troisième section du chapitre 4 de Mille plateaux affirme que la littérature est une «langue dans la langue». Comment comprendre cette thèse ?

Soit une liste arbitraire des auteurs que nous aimons... On remarque qu'ils sont plus ou moins dans la situation d'un bilinguisme: Kafka Juif tchèque écrivant en allemand, Beckett Irlandais écrivant à la fois en anglais et en français, Luca d'origine roumaine, Godard et sa volonté d'être suisse. Mais ce n'est qu'une occurrence, une occasion, et l'occasion peut être trouvée ailleurs. [...] Quand on fait subir aux éléments linguistiques un traitement de variation continue, quand on introduit dans le langage une pragmatique interne, on est forcément amenés à traiter de la même façon des éléments non linguistiques, gestes, instruments, comme si les deux aspects de la pragmatique se rejoignaient, sur la même ligne de variation, dans le même continuum ${ }^{8}$.

Ce passage révèle comment la littérature, pour Deleuze et Guattari, représente une mise en variation de la langue, la construction d'une langue dans la langue. Il ne s'agit pas du tchèque, de l'allemand, de l'irlandais ou

7. J. L. Borges, Ficciones, in Obras completas, t. I, Barcelone, Emecé, 1989-1996, p. 436 ; J. L. Borges et O. Ferrari, Diálogos, Barcelone, Seix Barral, 1992, p. 206. C'est nous qui traduisons.

8. $M M$, p. 123-124. 
du français, mais d'une langue majeure et d'une langue mineure ${ }^{9}$. C'est dans ce sens que le bilinguisme n'est qu'une occasion pour la création littéraire, comme il peut y en avoir autres. Â plusieurs reprises, Deleuze renverra à la thèse de Proust qui affirmait que les chefs-d'œuvre sont écrits dans une sorte de langue étrangère ${ }^{10}$. Mais il la fera sienne, en assimilant la langue étrangère à la langue mineure et la langue mère à la langue majeure. Écrire sera mettre la langue en variation, conduire la langue majeure vers sa forme mineure, introduire des différences dans une langue officielle. C'est à l'aide de ce schéma que Deleuze analysera plusieurs procédés concrets utilisés pour mettre la langue en variation : le «mot-passion » et le «mot-action» d'Artaud $^{11}$, les différents types de mots ésotériques de Lewis Carroll ${ }^{12}$, les répétitions de mots de Roussel et Péguy ${ }^{13}$, mais surtout la formule de Bartleby. "On dirait d'abord que la formule est comme la mauvaise traduction d'une langue étrangère. [...] Peut-être est-ce elle qui creuse dans la langue une sorte de langue étrangère ${ }^{14}$.

Mettre la langue en variation, construire une langue étrangère dans la langue, le «style» n'est pas autre chose. «Ce qu'on appelle style, qui peut être la chose la plus naturelle du monde, c'est précisément le procédé d'une variation continue $»^{15}$. Or à partir de cette conception, Deleuze déduira une fonction politique de la littérature. Comment le fait-il ? Une langue ne devient pas standard ou majeure spontanément. Sa nature spontanée consiste plutôt dans le mouvement, la variation, la différenciation. Si elle se stabilise comme langue majeure, c'est parce qu'une force politique y a intérêt. Cette force, c'est l'État. Soucieux de circonscrire un territoire, d'identifier une population, il a besoin, parmi plusieurs procédés, de la constitution d'une langue officielle; parmi plusieurs savoirs, d'une science linguistique ; et, parmi plusieurs institutions, d'une académie royale, par exemple. Ainsi, à partir du moment où la langue majeure est le produit d'une force politique, Deleuze comprend la mise en variation de cette langue, c'est-à-dire la langue

9. Les concepts de «majeur» et «mineur», chez Deleuze, sont d'ordre politique et non numérique; ils renvoient à une différence de pouvoir et non de quantité. "Minorité et majorité ne s'opposent pas d'une manière seulement quantitative. [...] La majorité suppose un état de pouvoir et de domination, et non l'inverse » $(M M, \mathrm{p} .133)$. La langue majeure est donc la langue dominante, la langue officielle; les langues mineures seront ses mutations et modifications, jargons ou patois. Or il ne faut pas croire qu'il s'agit d'une distinction entre deux langues parce que, d'un côté, une langue mineure n'échappe pas aux conditions d'un traitement qui en dégagerait constantes et universaux, et, de l'autre, plus une langue a ou acquiert les caractères d'une langue majeure, plus elle est travaillée par des variations continues qui la transposent en mineure. Donc «majeur» et «mineur» ne caractérisent pas deux sortes de langues, mais plutôt deux «usages» ou «fonctions » de la langue (MM, p.131).

10. Ibid., p. 124, par exemple.

11. $L S$, p. 110.

12. Ibid., p. 58-59.

13. DR, p. 34 .

14. $C C$, p. 92 .

15. $M M$, p. 123. 
mineure, comme une force contraire. D'où sa fonction politique. C'est donc parce que Deleuze pense la standardisation relativement à l'État et parce qu'il conçoit le style comme l'opération littéraire d'une mise en variation de la langue, qu'il affirmera souvent : «le style, c'est de la politique ${ }^{16}$.

L'activité littéraire ne mène pas une bataille seulement contre le troisième postulat de la linguistique selon lequel la langue serait un système homogène, mais également contre le premier, selon lequel le langage serait informatif $^{17}$. Introduire une pragmatique interne dans le langage ${ }^{18}$, qu'est-ce que cela veut dire ? Aussi bien dans Mille plateaux que dans Critique et clinique ou le livre sur Kafka, Deleuze dira à chaque fois que quand la langue est mise en variation, c'est tout le langage qui tend vers sa limite. "C'est comme si trois opérations s'enchaînaient : un certain traitement de la langue ; le résultat de ce traitement, qui tend à constituer dans la langue une langue originale ; et l'effet, qui consiste à entraîner tout le langage, à le faire fuir, à le pousser à sa limite propre pour en découvrir le Dehors, silence ou musique $\gg^{19}$. Quand la littérature fait naître une langue dans la langue, le langage tout entier tend vers sa limite. Quelle est cette limite ? C'est celle qui établit le premier postulat : la communication, la signification, la désignation. La parole devient une activité parmi les activités, voilà quel est le sens d'insérer une pragmatique interne à la langue et de faire un usage non signifiant de celle-ci, de traiter les éléments linguistiques comme des éléments non linguistiques, gestes ou instruments. Quand Deleuze affirme que l'écrivain fait bégayer, murmurer ou piauler la langue ${ }^{20}$, il indique que la langue est devenue une forme d'expression non significative. Il ne s'agit pas sans doute d'un bégaiement au sens littéral, d'une répétition des syllabes tout au long de la page. Mais il ne s'agit pas non plus d'une métaphore : c'est, non pas ce que l'écrivain dit, mais ce qu'il fait - ce qu'il fait lorsqu'il dit. Qu'en est-il, ensuite, du statut du silence ? Comment peut-il s'identifier à la musique, alors qu'il est plutôt son contraire ? Même si Deleuze parle d'un dehors, la limite qu'il trace dans ce cas est intérieure au langage. C'est la limite qui sépare la fonction significative de la fonction performative. La musique se confond avec le silence non parce qu'elle serait extralinguistique, mais parce que la parole devient une expression non référentielle, parce que le langage, précédemment, a été identifié à la communication.

\section{ID, p. 397. Cf. aussi p. 354.}

17. Voilà respectivement les premier et troisième postulats de linguistique, selon Deleuze et Guattari : «Le langage serait informatif, et communicatif» $(M M$, p. 95) ; «Il y aurait des constantes ou des universaux de la langue, qui permettraient de définir celle-ci comme un système homogène » (Ibid., p. 116). Les verbes au conditionnel permettent de déduire quelles sont les thèses que Deleuze et Guattari prétendent soutenir : le langage n'est ni informatif ni communicatif, mais plutôt performatif ; la langue est un système hétérogène en mouvement continu.

18. Cf. la citation qui correspond à la note 8 .

19. CC, p. 93-94. Cf. aussi p. 73 ; $M M$, p. $137 ; K$, p. 49.

20. $C C$, p. 135 . 
Enfin, à la mise en variation de la langue et à la tension du langage vers sa limite s'ajoutera ensuite une troisième fonction politique de la littérature, qui était déjà présente dans la Présentation de Sacher-Masoch, mais qui parviendra à son plein développement dans le livre portant «sur la littérature en général », Critique et clinique. En 1967, Deleuze dénonçait déjà la critique psychanalytique consistant à considérer les auteurs comme des malades. Commençant par une question qui rappelle l'orientation pragmatique et politique de sa philosophie de l'art - « À quoi sert la littérature ? $»^{21}-$, il répond que, dans les cas de Sade et Masoch, du moins, elle sert à désigner des perversions. Ainsi, les auteurs deviennent moins les malades que les médecins des civilisations, les symptomatologues plus précisément. L'antécédent de cette réflexion est sans doute Nietzsche, qui se proclamait psychologue de la culture. Le point d'aboutissement, il faut le chercher dans le livre de 1993 : «L'écrivain comme tel n'est-il pas malade, mais plutôt médecin, médecin de soi-même et du monde ? ${ }^{22}$. La littérature donc, quant à sa fonction politique, ne se limite pas à mettre la langue en variation, mais elle se constitue aussi comme diagnostic de ce qui affecte l'homme, en isolant les symptômes et en désignant les cadres.

L'art littéraire prend donc le contre-pied de la linguistique que critiquent Deleuze et Guattari. La philosophie du langage de Deleuze et Guattari a en effet comme but la dénonciation d'une linguistique qui, en séparant la langue des autres pratiques sociales, en la constituant comme un système homogène et en considérant le langage comme informatif ou représentatif, s'autoproclamerait comme une science neutre ${ }^{23}$. Visant la défense de la diversité linguistique, Deleuze et Guattari sont amenés, tout d'abord, à assumer le caractère intéressé de tout savoir ; ensuite à promouvoir une linguistique qui considère la langue en variation continue, comme une pratique parmi les pratiques, et le langage dans sa capacité non représentative, mais constitutive du réel. C'est en fonction de ces thèses que sera pensée la littérature. Or à partir du moment où, comme Bergson ${ }^{24}$, Deleuze considère que le philosophe est un écrivain, toutes les caractéristiques de la littérature vont passer du côté du discours philosophique. Celui-ci, en effet, manifestera une modalité propre pour

\section{PSM, p. 13.}

22. $C C$, p. 14.

23. Voilà respectivement les deuxième et quatrième postulats : «Il y aurait une machine abstraite de la langue, qui ne ferait appel à aucun facteur "extrinsèque" » $(M M$, p. 109) ; "On ne pourrait étudier scientifiquement la langue que sous les conditions d'une langue majeure ou standard » (ibid., p. 127). Une fois de plus, l'usage du conditionnel suggère les critiques correspondantes de Deleuze et Guattari : il y a une interpénétration de la langue, du champ social et des problèmes politiques (ibid., p. 115-116) ; une considération rigoureuse de la langue qui se soustrait aux conditions d'une langue majeure et standard entraîne une réforme épistémologique, c'est-à-dire l'abandon de la conception de la science comme détermination désintéressée de constantes et universaux (ibid., p. 127-139).

24. «Le philosophe peut n'être pas musicien, mais il est généralement écrivain » (H. Bergson, Les deux sources de la morale et de la religion, Paris, PUF, 2003, p. 268). 
mettre la langue en variation, fera lui aussi tendre le langage vers le silence, ou plutôt vers sa fonction performative, et se constituera comme une opération de diagnostic de la culture. D'où la légitimité d'une assimilation de la philosophie à la littérature.

\section{La philosophie comme branche de la littérature}

Tout d'abord, la philosophie, de la même façon que la littérature, construit une langue dans la langue. "Le baptême du concept sollicite un goût proprement philosophique qui procède avec violence ou avec insinuation, et qui constitue dans la langue une langue de la philosophie, non seulement un vocabulaire, mais une syntaxe atteignant au sublime ou à une grande beauté ${ }^{25}$. On remarque comment Qu'est-ce que la philosophie? utilise les thèses précédentes en domaine métaphilosophique. Le problème consiste alors à comprendre d'abord quel type de langue la philosophie construit dans la langue, ce qu'est une langue philosophique, et en quoi elle diffère de celle qu'une littérature construit. Il s'agira ensuite d'expliquer pourquoi cette définition de la philosophie dans les mêmes termes que la littérature ne rapproche pas la conception deleuzienne de celle de Borges.

Pourquoi une philosophie est-elle une langue dans la langue ? Pourquoi l'activité philosophique met-elle la langue en variation de façon à construire une expression particulière ? La réponse générale et pré-philosophique à ces questions est la même que celle qu'on pourrait donner si on demandait pourquoi Deleuze pense l'histoire de la philosophie comme collage, thêâtre ou art du portrait : il s'agit de faire quelque chose de nouveau avec des vieux éléments, quelque chose de différent avec des éléments déjà donnés. À ceci près que dans ce cas, les vieux éléments seront les mots du langage ordinaire, et non les philosophies de la tradition, comme dans le cas de l'histoire ; la différence sera donnée dans les mots du nouveau discours philosophique, et non dans la création de concepts ; et l'action consistera dans une parole, dans un certain emploi du langage. En effet, lorsqu'un concept sera créé, il sera inexprimable par la langue dans toutes ces variations, et cette création réclamera une variation de plus. C'est que, en fait, le concept et le mot n'ont pas des fonctions différentes par rapport au réel. Dans la théorie du concept qui se développe dans Qu'est-ce que la philosophie?, Deleuze nous dit que celui-ci ordonne le chaos virtuel en lui donnant une consistance. Or à partir du moment où ce chaos est identifié à une instance antérieure à l'opération des principes d'association ${ }^{26}$, et à partir du moment où il est décrit de la même façon que dans Empirisme et subjectivité est décrite cette instance, à savoir comme un état où on ne 
peut pas suivre nos idées, un état où elles naissent et s'évanouissent trop rapidement $^{27}$, nous pouvons conclure que le concept a une fonction constitutive par rapport au réel. Si Deleuze insiste sur le fait que le concept ne représente pas le réel et ne réfère pas à un état de choses ${ }^{28}$, s'il déclare qu'il est ce même réel ${ }^{29}$, c'est justement parce qu'il confère au concept une telle fonction. Or que nous dit-il du mot d'ordre dans la critique du premier postulat de la linguistique ? Justement que le mot n'est pas informatif ou communicatif, que sa fonction n'est pas la signification ou représentation du réel, mais une certaine intervention. «Il semble que ces actes [les actes du langage] se définissent par l'ensemble des transformations incorporelles ayant cours dans une société donnée, et s'attribuent aux corps de cette société $»^{30}$. «En exprimant l'attribut non corporel, et du même coup, en l'attribuant au corps, on ne représente pas, on ne réfère pas, on intervient en quelque sorte, et c'est un acte de langage ${ }^{31}$. Le concept et le mot ne s'identifient pas seulement négativement à partir de ce qu'ils ne sont pas (une représentation du réel), mais surtout positivement à partir de leur fonction commune : une mise en ordre ${ }^{32}$, une constitution du réel ${ }^{33}$. Ce n'est donc pas un hasard si les expressions pour caractériser l'activité de l'un et l'autre correspondent à un même champ lexical (le mot intervient, le concept découpe et recoupe ${ }^{34}$ ); c'est l'indice d'une communauté de fonction. Mot d'ordre et concept ont donc tous les deux une fonction constitutive du réel, ce qui signifie à la fois que tout discours suppose une philosophie, ou au moins une ontologie, et que toute création de concepts, en tant qu'elle procède à un découpage et un recoupement, à une reconfiguration de l'ontologie, à une réorganisation ou un réagencement, implique un exercice poétique, une mise en variation de la langue. John Rajchman l'énonce de la façon suivante : «En philosophie, le style est précisément un moyen pour rétrécir le langage de façon à atteindre les concepts qui lui sont particuliers ${ }^{35}$. Effectivement, si le style n'est rien d'autre que la mise en

27. Ibid., p. 45, p. 111 , p. 189 , p. 196 ; ES, p. 92.

28. $Q P h$, p. 16, p. 27, p. 137.

29. Ibid., p. 137.

30. $M M$, p. 102.

31. Ibid., p. 110.

32. « Nous demandons seulement un peu d'ordre », déclare Deleuze dans la première phrase de la conclusion de Qu'est ce que la philosophie ?, en faisant allusion à l'activité du concept ; «l'unité élémentaire du langage - l'énoncé -, c'est le mot d'ordre », affirme la réponse au premier postulat de la linguistique, renvoyant à un ordre qui n'est pas seulement commandement politique, mais aussi ordonnancement ontologique.

33. Il serait possible de poursuivre l'argumentation pour montrer que, en plus, le réel constitué, dans les deux cas, est le même : un réel virtuel ou incorporel (cf. $Q P h, p .112$, pour le cas du concept, et $M M$, p. 102 et p. 110, pour le cas du mot) et, plus précisément, l'événement (cf. $Q P h$, p. 36, pour le concept, et $M M$, p. 109, pour le mot).

34. $Q P h$, p. 21.

35. J. Rajchman, The Deleuze connections, Cambridge-London, The MIT Press, 2000, p. 117.

C'est nous qui traduisons. 
variation de la langue et qu'à ceci même procède la philosophie, il est bien cohérent de concevoir un style proprement philosophique.

«Les grands philosophes sont aussi des grands stylistes. [...] Le style, c'est une mise en variation de la langue... Pour cela, il faut que le langage ne soit pas un système homogène, mais un déséquilibre, toujours hétérogène... ${ }^{36}$. Quelle différence y a-t-il alors entre le style littéraire et le style philosophique ? Lorsque Deleuze affirme, plus polémique que jamais, que les grands philosophes sont des grands stylistes, comment ne pas confondre son propos avec un esthétisme?

Bien que le vocabulaire en philosophie fasse partie du style, parce qu'il implique tantôt l'invocation de mots nouveaux, tantôt la valorisation insolite de mots ordinaires, le style est toujours affaire de syntaxe. Mais la syntaxe est un état de tension vers quelque chose qui n'est pas syntaxique ni même langagier (un dehors du langage). En philosophie, la syntaxe est tendue vers le mouvement du concept ${ }^{37}$.

Le style implique toujours la même chose : une variation de la langue. Or, en philosophie cette variation trouve une raison d'être précise et qui n'est pas d'ordre esthétique : le mouvement du concept. Le philosophe ne crée ni des mots extraordinaires ni ne confère un nouveau sens aux mots ordinaires arbitrairement ou pour embellir ou obscurcir son discours. Le philosophe met la langue en variation seulement quand celle-ci ne parvient pas à exprimer une nouveauté conceptuelle. Cela ne veut pas dire que le style soit gratuit en littérature. Dans ce cas, il sera subordonné à la création d'affects et de percepts. Le besoin d'exprimer des nouvelles manières de percevoir et de sentir conduit l'écrivain à forger la langue ; c'est l'innovation conceptuelle, la reconstitution de la dimension virtuelle du réel qui guide le philosophe.

Du moment où le discours philosophique est considéré comme langue dans la langue, comme littérature, la force politique de celle-ci lui sera transmise. Les deux tendances du langage que la littérature exacerbait étaient son caractère performatif et sa variation continue. On vient de voir premièrement comment le discours philosophique lui aussi met la langue en variation. Or, deuxièmement, puisque la production d'une langue dans la langue pousse simultanément le langage tout entier vers sa limite, la parole philosophique elle aussi est conçue comme une parole performative. C'est ce que Deleuze dit de l'aphorisme chez Nietzsche : "C'est la relation avec le dehors ${ }^{38}$; et de son propre ouvrage, L'anti-CEdipe : «Il s'agit de voir s'il fonctionne, et comment, et pour qui. C'est lui-même une machine. Il ne s'agit pas de le relire, il faudra faire autre chose $»^{39}$. En fait, cette force

36. PP, p. 192. Cf. aussi p. 223.

37. Ibid., p. 223. Cf. aussi p. 192 : «Le style en philosophie, c'est le mouvement du concept $»$.

38. ID, p. 355.

39. $P$ P, p. 36. Cf. aussi p. 17. 
performative du discours philosophique pouvait se déduire de sa capacité constitutive, la fonction de produire le réel.

Enfin, c'est le troisième aspect de la force politique de la littérature que la parole du philosophe recueillera, à savoir la fonction clinique : « La philosophie n'a pas pour objet de contempler l'éternel, ni de réfléchir l'histoire, mais de diagnostiquer nos devenirs actuels ${ }^{40}$. Or dans quelle mesure cette activité est-elle proprement discursive ? À titre d'exemple, on peut penser à ce que Deleuze dit de l'écriture avec Guattari. Cela a eu « une fonction précise », déclare-t-il.

Une chose très choquante dans les livres de psychiatrie ou même de psychanalyse, c'est la dualité qui les traverse, entre ce que dit un malade supposé et ce que dit le soignant sur le malade. Entre le «cas» et le commentaire ou l'analyse du cas. [...] Cela permet tous les écrasements de ce que dit le malade... Nous, on n'a pas prétendu faire un livre de fou, mais faire un livre où l'on ne savait plus, où il n'y avait plus lieu de savoir qui parlait au juste, un soignant, un soigné, un malade présent, passé ou à venir ${ }^{41}$.

Voilà comment c'est un fait discursif, l'écriture à deux, qui achève la nouvelle relation entre critique et clinique, qui conserve la fonction diagnosticienne de la philosophie, en faisant sauter en même temps l'identification de l'auteur avec le malade.

Les considérations de Deleuze sur le discours philosophique supposent bien sa philosophie de la littérature. L'un et l'autre auront les mêmes fonctions. Or ceci, loin de nous orienter dans la direction d'un esthétisme, nous permet d'y échapper, parce que ces fonctions sont, en principe, politiques: arracher la langue à l'opération de normalisation de la linguistique, conférer au langage une force performative, assigner au discours une capacité de diagnostic. Ce n'est donc pas une différence au sujet de la philosophie qui distingue Deleuze de Borges, puisqu'ils la considèrent tous les deux comme branche de la littérature. C'est plutôt une différence dans la façon de concevoir la littérature. Qu'est-ce que suppose en effet, la variation philosophique de la langue ? Si la langue, en philosophie, est mise en variation, c'est parce que de nouveaux concepts sont créés. Â un nouveau concept correspondra un nouveau mot, un vieux mot à un nouveau sens, une nouvelle syntaxe. Pour l'instant, on dira donc que $l a$ création conceptuelle implique un travail littéraire. L'explicitation de la théorie générale du discours philosophique et l'examen du style proprement deleuzien permettront peut-être de préciser cette thèse de façon détaillée. Il est donc temps d'interroger les mécanismes concrets, de montrer comment le discours philosophique fait ce qu'on dit qu'il fait, et de déterminer quels sont les procédés qui, non seulement supposent une création de concepts, mais qui la permettent.

40. $Q P h$, p. $107-108$.

41. ID, p. 305. 


\section{Théorie générale du discours philosophique}

Il y a tout d'abord une expression philosophique par excellence, pense Deleuze, une expression qui à elle seule constitue une langue dans la langue : «en tant que ».

S'il fallait définir la philosophie par un mot, on dirait que la philosophie c'est l'art du « en tant que ». [...] Pourquoi ? [...] Le «en tant que » renvoie à des distinctions dans le concept qui ne sont pas perceptibles dans les choses mêmes. Quand vous opérez par distinctions dans le concept et par le concept, vous pouvez dire : «la chose en tant que, c'est-à-dire l'aspect conceptuel de la chose $»^{42}$.

Pourquoi s'agit-il de l'expression philosophique par excellence ? Parce que c'est la voix du découpage et du recoupement. Du moment où on définit la philosophie comme une activité de constitution du réel, on affirme que nos concepts établissent entre les choses des nouvelles répartitions, entre les objets des nouvelles frontières, qui vont jusqu'à questionner l'organisation du réel à partir des objets ou des choses. "En tant que », c'est le tracé d'un périmètre, c'est le dessin d'un champ transcendantal qui a pour conséquence une certaine image de l'expérience : "des distinctions dans le concept qui ne sont pas perceptibles dans les choses mêmes », peut-on lire dans la citation précédente. Quand Deleuze explique ce qu'est l'Idée platonicienne, c'est dans ces termes qu'on doit le comprendre : «Qu'est ce qu'une Idée ? [...] C'est une chose qui ne serait pas autre chose... Une mère c'est une mère, mais elle n'est pas que mère... Elle est par exemple épouse, et puis elle est elle-même fille d'une mère... Supposons une mère qui ne soit que mère... C'est cela qu'il faut appeler alors "Idée de mère" ${ }^{43}$. On pourrait bien dire : «c'est en tant que mère qu'une femme... ». «En tant que », c'est la pureté du concept. On prend une certaine réalité précédemment constituée comme femme, et sur cette réalité on recoupe une nouvelle forme, la mère. Donc voilà un premier trait discursif de la philosophie en tant que constitution du réel. Voilà une expression motivée non pas par tel ou tel concept, mais par le concept dans sa généralité.

Or quand on a affaire à un tel concept, on se trouve face au problème de sa nomination. De quelles options disposons-nous quand nous construisons une langue philosophique dans la langue ? «D'abord les concepts sont et restent signés, substance d'Aristote, cogito de Descartes, monade de Leibniz... Mais aussi certains réclament un mot extraordinaire, parfois barbare ou choquant, qui doit les désigner, tandis que d'autres se contentent d'un mot courant très ordinaire... Certains sollicitent des archaïsmes, d'autres des néologismes... » ${ }^{44}$. Barbarismes, néologismes, archaïsmes sont

42. Cours du 24 mars 1981, archives sonores, source BNF, ou bien téléchargeable sur http://www.webdeleuze.com/php/texte.php?cle=45\&groupe=Spinoza\&langue=1 .

43. $A B C$, Lettre $\mathrm{H}, 37: 30-39: 10$.

44. $Q P h$, p. 13 . 
quelques stratégies de nomination conceptuelle. Or s'agit-il strictement d'une nomination, d'une désignation, ou d'un baptême? La relation semblerait accorder une certaine primauté au concept, une certaine antériorité, comme si le mot venait après coup pour l'indexer. Cependant, le langage ordinaire a lui aussi une force constitutive et suppose, dans ce sens, une philosophie sur laquelle la philosophie vient faire ses découpages et recoupements. Ainsi, la création conceptuelle ne doit pas être envisagée comme un exercice logique muet, après lequel le philosophe irait charger dans un sac les mots plus ou moins arbitrairement, mais comme une activité dans la langue et contre la langue, comme une activité en elle-même linguistique. Jean-Luc Nancy affirme ainsi de la philosophie de Deleuze :

C'est une philosophie de la nomination et non du discours. [...] $\mathrm{La}$ nomination elle-même n'est pas une opération sémantique : il ne s'agit pas de signifier les choses, il s'agit plutôt d'indexer par noms propres les éléments de l'univers virtuel. Aucune philosophie ne fait peut-être un tel usage des noms propres : d'une part elle imprime un «devenir-concept» à des noms propres [...], d'autre part elle imprime un «devenir-nom-propre » à des concepts. [...] La nomination est donc plutôt un geste matériel : le mouvement pour déplacer une masse, une charge, un tracé, pour l'indexer autrement. "Porter au langage », cela ne signifie pas ici traduire en langage [...], mais cela signifie faire porter par le langage le poids de ce qui n'est pas lui. L'incorporel chargé du corporel: non pas d'en donner ni d'en manifester un sens, mais de l'effectuer autrement ${ }^{45}$.

S'il arrive que Nancy s'exprime en termes de nomination - Deleuze luimême le fait -, on s'aperçoit qu'il la conçoit non pas comme traduction mais comme effectuation. Il y a un devenir-nom du concept autant qu'un devenirconcept du nom : autant dire que le nom est déjà concept et le concept déjà nom. Voici le point le plus important par rapport à notre problème général : le concept ne se construit que par un remaniement linguistique de la langue ${ }^{46}$. C'est en ce sens que la création du concept et la mise en variation philosophique de la langue sont les deux faces d'un même phénomène. Le discours philosophique n'est ni une mise en forme du concept, ni la possibilité de sa transmission, ni sa nomination, ni un ornement. Le discours

45. J.-L. Nancy, «Pli deleuzien de la pensée », in Gilles Deleuze. Une vie philosophique, Éric Alliez (dir.), Paris, Synthelabo, 1996, p. 120.

46. Dans une version précédente de l'Introduction à Qu'est-ce que la philosophie ?, il est possible d'observer que Deleuze a barré trois fois sur la même page le terme «nom » et écrit au-dessus «mot»: «les concepts sont et restent signés, substance d'Aristote, cogito de Descartes... Mais aussi certains réclament un nom mot extraordinaire, parfois barbare ou choquant »; «tandis que d'autres se contentent d'un mot courant très ordinaire... »; « il doit y avoir dans chaque cas une étrange nécessité de ces mots et de leur choix, comme élément de style » (archives IMEC, fonds Félix Guattari, manuscrit : "Introduction : ainsi donc la question... », cote GTR2. Aa-07.02.01). Le fait que le terme soit remplacé trois fois prouve que ce n'est pas une simple question de rhétorique. Le sens du remplacement, pour sa part, peut bien être interprété comme une mise à distance d'une relation de nomination ou désignation. 
philosophique est le lieu et la matière de la création conceptuelle : c'est dans le langage, contre la langue, et avec les mots qu'on crée des concepts.

Néologismes, barbarismes, archaïsmes sont donc différentes stratégies de signature. Or, qui signe ? Quelle est l'instance énonciative en philosophie? De même que Blanchot ${ }^{47}$, Foucault ${ }^{48}$ ou le poète italien Ferlinghetti $^{49}$, Deleuze va soustraire l'énonciation littéraire en général ${ }^{50}$ et philosophique en particulier à la première personne : "Qui est Je ?, c'est toujours une troisième personne $»^{51}$. Nous dirons que, en ce sens, il fait fonctionner dans le domaine esthétique une critique qui déborde amplement ce domaine, celle qui vise la subjectivité comme origine de l'expérience. $\mathrm{Si}$ entre le concept et le plan d'immanence, Deleuze place le personnage conceptuel comme troisième élément de la philosophie, c'est justement pour assigner à l'énonciation une instance non subjective. "Dans l'énonciation philosophique, on ne fait pas quelque chose en le disant, mais on fait le mouvement en le pensant, par l'intermédiaire d'un personnage conceptuel. Aussi les personnages conceptuels sont-ils les vrais agents d'énonciation $»^{52}$. Or, si le personnage conceptuel est une figure qui montre comment l'énonciation philosophique n'est pas personnelle, il n'est pas le seul. Même le nom propre est considéré comme l'expression d'une œuvre, d'une pensée, plutôt que d'un auteur. C'est ce que l'on voit lors de la distinction entre la fonction du nom propre en science et en philosophie : «les noms propres marquent dans un cas une juxtaposition de références, et dans l'autre une superposition de feuillets $»^{53}$. Or, si d'un côté Deleuze cherche à échapper à la personnalisation de l'énonciation, au «narcissisme » de la notion d'auteur $^{54}$, à la compréhension psychologique ou psychanalytique de l'expression, de l'autre, il ne peut abandonner l'individualité, la singularité que toute création en tant que telle exige pour son instance énonciative. Ainsi, Deleuze semble être dans une position ambigüe par rapport au romantisme : d'une part, il va adopter la figure du génie pour rendre compte de l'originalité de l'œuvre ; mais d'autre part, il va l'épurer de son caractère subjectif. Comment concilier donc la critique de la subjectivité et la figure du génie ? Avec une instance énonciative que Deleuze appellera « singularité pré-individuelle» ou «impersonnelle». Avec cette notion, il prétend assigner aux énoncés philosophiques, d'une part, la même universalité, la même nécessité, la même objectivité qu'on attribue normalement aux

47. $C C$, p. 13.

48. $F$, p. $62-63$.

49. $L S$, p. 125 ; ID, p. 199.

50. «Écrire n'est pas raconter ses souvenirs, ses voyages, ses amours et ses deuils, ses rêves et ses fantômes...» $(C C$, p. 12).

51. $Q P h$, p. 63.

52. $Q P h$, p. 63.

53. $Q P h$, p. 122. Rappelons que, selon Deleuze, l'histoire de la philosophie n'est pas succession de systèmes mais superposition de plans, chaque plan comptant plusieurs feuillets.

54. D, p. 61 . 
énoncés de la science; et d'autre part, la même originalité, la même singularité, qu'on attribue normalement aux énoncés littéraires ou poétiques $^{55}$. C'est à cette singularité impersonnelle que pense Deleuze chaque fois qu'il s'interroge sur la fonction de la troisième personne du singulier, sur celle d'une quatrième parfois, sur celle du personnage conceptuel, celle du nom propre ou du pronom neutre par rapport à l'énonciation philosophique. L'enjeu est toujours le même : distinguer la singularité de cette énonciation aussi bien de l'expression d'un état d'âme personnel que de la reproduction mécanique d'une formule d'opinion.

La critique de la subjectivité parcourt dans l'œuvre de Deleuze la théorie du concept - le concept n'est pas représentation subjective mais une entité objective parmi les objets du monde -, la théorie de l'énonciation, comme on vient de le voir, et l'éthique, au sens où le projet libératoire n'est pas orienté vers la pleine réalisation des facultés du sage, mais par un devenir-autre - femme, animal ou molécule. Or la cohérence de cette critique n'est pas donnée seulement par son fonctionnement simultané dans des domaines divers, mais elle va plus loin. D'une part, c'est justement parce que le concept n'est pas une représentation subjective que l'instance de son énonciation ne l'est peut-être pas non plus. De même qu'une opération arithmétique ne concerne pas des dispositions personnelles, de même que toute théorie de l'inspiration en esthétique a comme effet une certaine dépossession du sujet, la théorie du concept, en tant que celui-ci se comprend comme chose parmi les choses, exige une énonciation non personnelle. D'autre part, la cohérence de la critique est donnée par le fait que l'écriture apparaît comme l'espace de réalisation du projet éthique. En effet, du moment où nous considérons que le je qui écrit est un autre, cet autre est le même qui règle le devenir de l'éthique. Deleuze l'affirme de la littérature en général ${ }^{56}$, et de l'écriture philosophique en particulier: « Devenir étranger à soi-même, et à sa propre langue et nation, n'est-ce pas le propre du philosophe et de la philosophie, leur style, ce qu'on appelle un charabia philosophique ? ${ }^{57}$. De cette façon, le discours philosophique ne possède pas une force politique seulement parce que sa parole est une action, ou parce qu'il met la langue en variation, ou encore parce qu'il dissout la dichotomie entre médecin et malade, afin de réaliser un diagnostic de la civilisation; sa force réside aussi dans le fait que son exercice est la réalisation même du projet éthique compris comme devenir-autre.

Voilà pour les considérations générales de Deleuze sur le discours philosophique qui concernent l'instance énonciative, le rapport du concept au mot et une expression précise - «en tant que ». C'est parce que Deleuze

55. On dit bien «qu'on attribue normalement » parce que la différence entre les énonciations philosophique, scientifique et artistique, selon Deleuze, ne joue pas ici, car elles sont toutes les trois aussi singulières (du point de vue de leur originalité) qu'objectives (du point de vue de leur statut).

56. $D$, p. 54 et p. $55 ; M M$, p. 10 , p. 229 , p. $293 ; K$, p. 14 , p. 63 ; $C C$, p. 11.

57. $Q P h$, p. $105-106$. 
comprend le concept comme une condition objective de la réalité, comme une fonction constitutive mais objective, que d'un côté, son énonciation ne renvoie pas à un sujet, mais à une troisième personne, et que de l'autre, l'expression si fréquemment mobilisée est «en tant que». Or ce qui intéresse notre propos, c'est surtout le deuxième point de la théorie. Dans la mesure où la relation du concept au mot n'est pas de nomination, il n'est plus possible d'affirmer que la création conceptuelle motive simplement un travail littéraire. Il semblerait plutôt qu'elle l'exige dans la mesure où c'est ce même travail qui construit le concept. Nous le déduisons surtout du fait que le langage, selon le premier postulat, a lui aussi une fonction constitutive. Mais plus concrètement, n'est-ce pas le concept de «concept» que Deleuze construit lorsqu'il développe ses réflexions sur l'instance énonciative et l'expression «en tant que »? Lire ces passages comme de la glose méta-discursive, c'est passer à côté de leur force et de leur pertinence. Ces passages ne constituent pas la mise en forme linguistique des concepts en question, mais leur formation même. C'est ainsi que la théorie du discours n'échappe pas à l'accusation d'esthétisme seulement parce qu'elle lui assigne une fonction politique, mais aussi parce qu'elle lui accorde une place dans la théorie de la connaissance. Il est vrai que la pensée deleuzienne ne vise pas tant la connaissance que l'expérience (au sens d'une aventure), et pas tant l'expérience (au sens kantien) que le monde. Disons donc tout simplement que le discours a autant une fonction pratique qu'épistémique.

Les considérations d'ordre général vont se compléter par certaines études de cas, qui concernent notamment le discours de Nietzsche (en particulier, la fonction de l'aphorisme), celui de Spinoza (sa célèbre théorie des deux ou trois Ethiques) et dans une moindre mesure ceux de Foucault, Heidegger et Kierkegaard. Dans chaque cas, ce qui est intéressant, c'est de remarquer comment Deleuze considère non seulement la forme du discours à partir d'une création conceptuelle qui la motive - aphorisme et expression du sens chez Nietzsche, les trois Ethiques et les trois genres de connaissance chez Spinoza -, mais comment il utilise le discours comme un instrument parmi d'autres pour montrer les innovations conceptuelles. Nous n'entrerons pas dans le détail de l'étude des cas - ce qui a déjà été fait ${ }^{58}$; mais nous privilégierons une analyse de la prose de Deleuze. Son intérêt consistera à dévoiler simultanément comment une création conceptuelle motive une certaine figure ou un certain procédé, et comment une réflexion sur la figure ou le procédé permet justement la création conceptuelle. Certains cas concerneront les problèmes généraux que Deleuze lui-même signale et que nous venons d'exposer. D'autres renverront à des problèmes intrinsèques à sa philosophie. Parmi les deux groupes, nous allons sélectionner seulement les plus polémiques.

58. Cf. M. Antonioli, Deleuze et l'histoire de la philosophie, Paris, Kimé, 1999, p. 93-109, pour le cas de Nietzsche ; P. Macherey, «Spinoza : une philosophie à plusieurs voix », in Philosophique, revue de la Faculté des Lettres de Besançon, Kimé, 1998 ; p. 5-22, pour le cas de Spinoza. 


\section{Le discours deleuzien}

Par rapport à la stratégie nominative, signalons tout simplement que si dans certains cas Deleuze choisit des néologismes (« on reproche parfois aux philosophes de créer des mots barbares... Déterritorialisation... C'est un mot dur à dire... C'est un très bon cas où un concept philosophique ne peut être désigné que par un mot qui n'existe pas encore... ${ }^{59}$ ), dans d'autres cas il se limite à employer des mots ordinaires avec un nouveau sens. C'est le cas du nom de l'être, largement analysé par Alain Badiou : vie $e^{60}$. Le problème de l'instance énonciative est un peu plus épineux, et se présente de différentes façons selon que l'on considère les monographies ou l'œuvre «en nom propre ». À propos de la deuxième, il ne s'agit pas simplement de rappeler qu'elle a été écrite en grande partie en collaboration, ni de montrer que Deleuze comprend cette même collaboration d'une façon particulière, de façon à gommer les limites de la notion d'auteur ${ }^{61}$. Ce qu'il faut montrer c'est comment cette stratégie conspire effectivement contre une instance subjective d'énonciation. Or, quelle est la différence entre travailler ensemble ou collaborer et travailler entre les deux ? La distinction renvoie à la méthodologie : les livres ne sont pas, en effet, des compilations d'articles où chacun signe le sien, mais le produit d'une correspondance et de réunions plus ou moins périodiques que Deleuze finalement rédigea ${ }^{62}$. C'est la décision d'une co-signature globale qui fonctionne comme une proclamation contre la notion d'auteur, et c'est une méthodologie de travail précise qu'incarne cette proclamation dans une pratique conséquente.

Or la question est encore plus délicate dans les cas des écrits dits « monographiques ». En général, on s'accorde à affirmer qu'ils sont écrits en discours indirect libre ${ }^{63}$. Qu'est-ce que ça veut dire ? Comme le discours indirect, cette figure renvoie l'énonciation à un deuxième agent. Mais dans sa forme libre, on dira en principe que cet agent demeure implicite dans

59. $A B C$, Lettre A, 13:50-14:45.

60. Cf. A. Badiou, «De la vie comme nom de l'être », in Gilles Deleuze. Immanence et vie, Paris, PUF, 2006, p. 29 ; Deleuze. «La clameur de l'Être », Paris, Hachette, 1997, p. 42-47.

61. «Travailler à deux, beaucoup de gens l'ont fait... Mais il n’y a pas de règle, de formule générale. [...] Nous n'étions que deux, mais ce qui comptait pour nous, c'était moins de travailler ensemble, que ce fait étrange de travailler entre les deux. On cessait d'être auteur. [...] On ne travaille pas ensemble, on travaille entre les deux » (D, p. 24).

62. Pour la méthode d'écriture, se rapporter au prologue de la biographie de F. Dosse, Gilles Deleuze, Félix Guattari. Biographie croisée, Paris, La Découverte, 2007, p. 11-29.

63. François Zourabichvili a été le premier, à notre avis, à soutenir cette thèse (Deleuze. Une philosophie de l'événement, Paris, PUF, 1994, p. 5). L'ont suivi Alain Badiou (op. cit., p. 25 et p. 146), Alberto Navarro Casabona (Introducción al pensamiento estético de Gilles Deleuze, Valencia, Tirant Lo Blanch, 2001, p. 206), Eduardo Pellejero (Deleuze y la redefinición de la filosofía, Madrid, Morelia Editorial, 2007, p. 143), Stéfan Leclercq (« La réception posthume de l'œuvre de Gilles Deleuze », in Alain Beaulieu (dir.), Gilles Deleuze, héritage philosophique, p. 146). Manola Antonioli, de son côté, en même temps qu'elle s'inscrit dans cette lignée, repère déjà son existence (op.cit., p. 7). 
le texte. Philippe Mengue présente un exemple très clair emprunté à L'éducation sentimentale : "Le domestique revint : "Madame allait recevoir Monsieur" » ${ }^{64}$. Qui dit «Madame» ou «Monsieur»? Sans doute pas le même narrateur qui dit «Le domestique revint». C'est la voix du domestique lui-même introduit dans le discours du narrateur. Or cette insertion ne se fait pas sous la forme du discours direct (Le domestique dit : «...»), ni sous la forme du discours indirect tout court (Le domestique dit que...), mais sous la forme du discours indirect libre. On ne dira pas simplement qu'un agent demeure implicite dans l'énonciation ; c'est plutôt que les agents deviennent indiscernables. En effet, il n'est pas tout à fait correct de dire que le domestique dit «Madame» ou «Monsieur». C'est plutôt le narrateur qui parle avec la voix du domestique ou bien le domestique qui parle à travers le narrateur. En tout cas - et c'est ça qui intéresse Deleuze - on ne peut plus assigner l'énonciation à un agent déterminé, puisqu'elle dépend d'un «agencement collectif». On comprend dès lors pourquoi les monographies seraient écrites en discours indirect libre. On assisterait dans ces textes à une dissolution du sujet qui commente et du sujet commenté. D'où les questions à propos des livres du type «Est-ce du Deleuze ? Est-ce du Spinoza?», «Est-ce du Deleuze ? Est-ce du Nietzsche ? » Or, de même que pour les écrits en nom propre, il ne suffisait pas de rappeler les remarques de Deleuze au sujet de l'écriture à deux, il ne suffit pas à présent de relier cette figure de prédilection de Deleuze aux monographies. Quasiment aucun commentateur ne donne d'exemple. Et lorsqu'ils le font, il n'est pas sûr que ce soit l'exemple adéquat. C'est le cas de Alain Badiou, qui écrit :

Si je lis par exemple : «force parmi les forces, l'homme ne plie pas les forces qui le composent sans que le dehors ne se plie lui-même, ne creuse un Soi dans l'homme » $(F ., 121)$, s'agit-il vraiment d'un énoncé de Foucault ? Ou déjà d'une interprétation? Ou est-ce tout simplement une thèse de Deleuze, puisqu'on y reconnait sa lecture de Nietzsche (le jeu de forces actives et réactives compose typologiquement l'homme), et qu'on y pointe un concept majeur de son œuvre terminale, celui de pli ?

Mais Badiou ne prend pas une phrase de Deleuze où il y aurait des éléments de Foucault, ou des termes de Foucault chez Deleuze. Il prend une phrase de Deleuze - certes, lisant Foucault - et il signale des termes qui viennent de Deleuze lui-même. Dans quel sens y aurait-il donc deux instances énonciatives ? Ce qu'il faut dévoiler, au contraire, c'est comment, dans des phrases de Deleuze, il y a des éléments qui viennent d'ailleurs, en particulier de l'auteur commenté. En ce sens, dans l'exemple de Badiou, le discours indirect libre concernerait plutôt la relation entre Deleuze et Nietzsche. Comment trouver un bon exemple alors? Il faut écouter les phrases d'autres auteurs que Deleuze, justement, fait siennes. Il y a un cas aussi notoire que récurrent: personne ne sait ce que peut un corps. Nous 
savons l'importance que, pour Deleuze, revêt la formule depuis les commentaires sur Spinoza $^{65}$. Or dans Dialogues, on lit : «Voilà la question : qu'est-ce que peut un corps? Expérimentez, mais il faut beaucoup de prudence pour expérimenter. Nous vivons dans un monde plutôt désagréable... ${ }^{66}$. C'est Deleuze qui parle, mais avec la voix de Spinoza. Ou bien Spinoza, mais par la bouche de Deleuze. Dans Dialogues, qui est plutôt le monologue d'un agent collectif, la phrase est devenue, non pas propriété de Deleuze, mais patrimoine universel. La même étude pourrait être poursuivie avec toutes les formules que Deleuze incorpore dans son corpus : celle de Proust, que nous avons déjà rencontrée, les beaux livres sont écrits dans une sorte de langue étrangère, ou une aussi brève que simple, Bonjour Théétète, que Deleuze, sans guillemets ni deux points, utilise pour faire parler l'image dogmatique de la pensée, aussi bien dans Différence et répétition ${ }^{67}$ que dans Qu'est-ce que la philosophie? ${ }^{68}$ On dira que tous nos exemples appartiennent à l'œuvre propre, et non pas aux monographies. C'est que, effectivement, l'examen suppose une thèse par rapport à la périodisation de l'œuvre. La distinction entre « histoire de la philosophie » et «philosophie» ne concerne pas des livres concrets, mais c'est une distinction idéale qui traverse de différentes façons les œuvres concrètes. Le discours indirect libre est l'expression non pas d'un ensemble de livres mais d'une certaine historiographie, présente aussi bien dans les monographies que dans les œuvres propres, à des degrés divers. Or qu'est-ce que cela veut dire, qu'il en est «l'expression»? Certainement pas seulement que Deleuze a choisi une forme de discours après s'être donné une conception de l'instance énonciative. C'est simultanément à travers la réflexion sur la figure et son emploi, comme à travers la réflexion sur l'écriture à deux et la stratégie de signature, que la conception de l'instance énonciative est partiellement construite.

Considérons maintenant les procédés destinés non pas à résoudre les problèmes que tout discours philosophique suppose, mais les problèmes intrinsèques à la philosophie deleuzienne. Nous pensons par exemple aux pseudo-métaphores, à l'anexactitude ${ }^{69}$, à la construction du type «article indéfini + nom propre + verbe infinitif ${ }^{70}$, au style télégraphique et à la phrase nominale ${ }^{71}$, à l'utilisation de la conjonction $e t^{72}$ ou à la substantivation de l'adjectif multiple ${ }^{73}$. Lorsque Deleuze affirme, par exemple, que l'événement est ce qui s'énonce selon des articles indéfinis,

65. SPE, p. 208 ; SPP, p. 28.

66. $D$, p. $75-76$.

67. DR, p. 176 et p. 181.

68. $Q P h$, p. 132.

69. $M M$, p. 31 et p. $454 ; P P$, p. $44-45$.

70. $M M$, p. 322.

71. $P P$, p. $52 ; D$, p. 78.

72. $D$, p. $70-72$.

73. $D R$, p. 236 ; $P P$, p. 23 ; $A O$, p. $50 ; M M$, p. $10 ; D$, p. 71. 
des verbes à l'infinitif, des noms propres, c'est le concept même d' «événement » qui se construit comme une singularité non individuelle ou impersonnelle. La création de concepts consiste dans cet exercice même de polissage des mots, de travail sur les sens ordinaires - qu'ils appartiennent au sens commun ou à la tradition philosophique. C'est pourquoi nous disons que la création de concepts survient dans le langage et contre la langue ; et que la désignation se confond avec l'avènement, le baptême avec la gestation.

Rentrons tout de même dans le détail d'un cas particulier, celui de l'expression au sujet de laquelle Deleuze affirme continûment «elle n'est pas métaphorique ${ }^{74}$. Quel est le sens de cet avertissement ? Prenons comme exemple le cas du devenir-animal. «Le devenir-animal n'a rien de métaphorique» affirme Deleuze ${ }^{75}$. La déclaration est certainement provocatrice, puisqu'elle ne se soucie pas de signaler l'inverse, à savoir, que l'expression devenir-animal ne doit pas se comprendre littéralement. En fait, Deleuze ira même jusqu'à soutenir qu'il faut comprendre l'expression à la lettre. Or ce que le lecteur doit se demander, c'est ce que Deleuze comprend par «métaphore » dans un cas, par «littéralité » dans l'autre. Deleuze ne veut pas que l'on prenne l'expression comme une métaphore en raison de tout ce qu'elle introduirait de fictif ou d'imaginaire dans le procès réel. Mais d'un autre côté, sans doute le devenir-animal ne doit pas se comprendre comme une mutation d'ordre biologique. Ou bien, en tout cas, cela dépend de la manière de comprendre la biologie, de quelle vie il s'agit. Ainsi nous sommes renvoyés de terme en terme à un langage qui, comme le croyait Bergson, semble plus adapté aux besoins de la physique qu'à la spéculation métaphysique. Au fond, le problème qu'avait Bergson par rapport au langage est le même que Deleuze dans ce cas, et il peut se résumer ainsi : comment exprimer l'esprit? Bergson le résout en distinguant des chaînes lexicales (celles de l'espace, de l'homogène, du divisible, de la simultanéité, de la matière d'un côté; de l'esprit, de la succession, du continu, de l'hétérogène de l'autre) et en soutenant que, strictement, la durée ne peut s'exprimer que métaphoriquement. La stratégie de Deleuze, même si son objectif est le même, sera inverse : utiliser un vocabulaire le plus physicaliste possible, emprunter des termes à l'optique, à la chimie, à la biologie pour exprimer le virtuel, et en même temps, soutenir qu' 'il ne s'agit pas là de métaphores ». Le moléculaire, l'intensif, les vitesses, la température, les couleurs... s'attribuent toujours à un ordre réel mais pas actuel, à un ordre incorporel, à une vie qui n'est pas la vie organique. "Réalité du deveniranimal, sans que l'on devienne animal en réalité », la formule exprime le problème et la crainte de tout empirisme supérieur, de tout empirisme qui élargit l'observable aux yeux de l'esprit: comment parvenir à exprimer le virtuel sans lui ôter sa teneur de réalité ? Bergson choisit la distinction et la

74. Zourabichvili présente une très grande liste d'exemples dans son Vocabulaire de Gilles Deleuze, Paris, Ellipses, 2003, p. 3.

75. K, p. 65. 
métaphore ; Deleuze, la confusion et son refus. C'est sans doute pourquoi Guy Lardreau affirme avec pertinence que «Deleuze, c'est du Bergson mal écrit, comme notre temps exigeait ${ }^{76}$. Soyons clairs : il n'est pas question de maîtrise rhétorique, mais de différences stylistiques. Et insistons : la stratégie littéraire n'est pas un choix arbitraire et second, mais elle renvoie à une différence dans la conception de l'esprit et de la langue qu'elle contribue à forger. Si Deleuze s'exprime en termes très concrets et ajoute qu'il ne parle pas métaphoriquement, c'est pour la même raison qu'il rebaptise l'esprit comme «virtuel»; il s'agit de lui donner une pleine réalité, voire une matérialité.

Quelle est la relation en philosophie entre le mot et le concept, quel est le rapport de la philosophie au langage, lorsqu'elle est conçue comme une création de concepts ? La théorie deleuzienne du discours philosophique que nous avons cherché à expliciter à partir de certaines remarques générales et certaines analyses de cas indique d'abord que toute création conceptuelle motive un travail littéraire. Or toute une réflexion sur les figures et les procédés révèle quelque chose de plus : loin d'être une simple mise en forme, un ornement ou la possibilité de sa communication, l'expression est un des instruments qui permet la création conceptuelle même, que ce soit à travers son simple emploi ou bien à travers une réflexion linguistique. C'est pourquoi la théorie du discours constitue de plein droit un moment de la théorie de la méthode.

À partir du moment où le concept aussi bien que le mot - qui sont deux aspects d'un même élément d'un certain point de vue - ont une fonction constitutive du réel, la conception deleuzienne du discours échappe au soupçon d'esthétisme. Certes, Deleuze s'efforce de penser le concept plutôt comme une expérience que comme une forme de connaissance. En effet, c'est un concept qui ne représente pas un état de choses extérieur, qui est susceptible d'une affectivité, etc. Mais sans doute, du moins, le discours aura une fonction épistémique. Cependant, le soupçon se dissout aussi grâce aux fonctions pratiques du discours philosophique : la mise en variation de la langue, le déplacement du langage vers sa limite, l'activité de diagnostic, la réalisation du but éthique. Deleuze, de même que Borges, pense jusqu'à un certain point la philosophie comme littérature. Mais l'équivalence, dans le cas du second, renferme tout son scepticisme gnoséologique et politique. C'est justement parce que les deux formes de scepticisme sont absentes chez le premier que l'équivalence ne conduit pas à l'esthétisme. "Branche de la littérature »? Certainement, mais ni dans le sens d'une dépendance vis-à-vis d'un tronc qui mettrait en danger la spécificité de la philosophie, ni dans le sens d'une activité ludique. «Branche de la littérature fantastique »? Oui encore : mais au sens où Deleuze interprète la déclaration de Foucault. " "Je n'ai jamais écrit que des fictions...". Mais jamais fiction n'a produit tant de

76. G. Lardreau, «L'histoire de la philosophie comme exercice différé de la philosophie (Deleuze historien) », in Gilles Deleuze. Immanence et vie, Paris, PUF, 2006, p. 67. 
vérité et de réalité ${ }^{77}$. Si l'affirmation de Borges peut s'appliquer à Deleuze, c'est au prix de plusieurs reformulations. De la littérature d'abord, qui cesse d'être une activité désintéressée ; de la fiction ensuite, qui n'est plus un voile entre l'homme et le monde, mais plutôt la constitution perspectiviste de ce dernier.

Est-ce que les philosophes écrivent mal en définitive comme l'affirme Lardreau à propos du style de Deleuze ou le confesse aussi Heidegger au paragraphe 7 d'Etre et temps ? Lardreau a le souci de remarquer tout de suite que cela «n'interdit nullement de [...] reconnaître [à Deleuze] un talent proprement littéraire ${ }^{78}$, et Heidegger justifie la lourdeur et l'absence de " grâce » de l'expression en remarquant que si on veut saisir l'étant en son être, et non pas simplement rendre compte de l'étant de façon narrative, ce ne sont pas seulement les mots qui manquent le plus souvent, mais avant tout la grammaire. Le sens métaphilosophique de sa confession est le même que celui de la conception deleuzienne du discours philosophique : le bien et le mal disparaissent au sein d'une beauté dont le critère est la nécessité. 


\section{Liste des abréviations :}

ABC. Deleuze, G., et Parnet, C., L'abécédaire de Gilles Deleuze, Paris, Editions Montparnasse, 2004.

AO. Deleuze, G., et Guattari, F., L'anti-CEdipe. Capitalisme et Schizophrénie 1, Paris, Minuit, 1972.

CC. Deleuze, G., Critique et clinique, Paris, Minuit, 1993.

D. Deleuze, G. et Parnet, C., Dialogues, Paris, Flammarion, 1977.

DR. Deleuze, G., Différence et répétition, Paris, PUF, 1968.

ES. Deleuze, G., Empirisme et subjectivité, Paris, PUF, 1953.

F. $\quad$ Deleuze, G., Foucault, Paris, Minuit, 1986.

ID. Deleuze, G., L'île déserte, Paris, Minuit, 2002.

K. Deleuze, G. et Guattari, F., Kafka. Pour une littérature mineure, Paris, Minuit, 1975.

LS. Deleuze, G., Logique du sens, Paris, Minuit, 1969.

MM. Deleuze, G. et Guattari, F., Mille plateaux. Capitalisme et Schizophrénie 2, Paris, Minuit, 1980.

PP. $\quad$ Deleuze, G., Pourparlers, Paris, Minuit, 1990.

PSM. Deleuze, G., Présentation de Sacher-Masoch, Paris, Minuit, 2004.

QPh. Deleuze, G. et Guattari, F., Qu'est-ce que la philosophie ?, Paris, Minuit, 1991.

SPE. Deleuze, G., Spinoza et le problème de l'expression, Paris, Minuit, 2003.

SPP. Deleuze, G., Spinoza. Philosophie pratique, Paris, Minuit, 1981 